\title{
BMJ Open 'Klar bleiben': a school-based alcohol prevention programme for German adolescents - study protocol for a cluster randomised controlled trial
}

\author{
Samuel Tomczyk, Reiner Hanewinkel, Barbara Isensee
}

To cite: Tomczyk S, Hanewinkel $R$, Isensee $B$. 'Klar bleiben': a school-based alcohol prevention programme for German adolescents-study protocol for a cluster randomised controlled trial. BMJ Open 2015;5:e010141.

doi:10.1136/bmjopen-2015010141

- Prepublication history for this paper is available online. To view these files please visit the journal online (http://dx.doi.org/10.1136/ bmjopen-2015-010141).

Received 30 September 2015 Accepted 22 October 2015

CrossMark

Institute for Therapy and Health Research (IFT-Nord), Kiel, Germany

Correspondence to Samuel Tomczyk; tomczyk@ift-nord.de

\section{ABSTRACT}

Introduction: There is a noticeable increase in hazardous alcohol use during adolescence, which is significantly associated with adverse consequences. In Germany, up to $30 \%$ of adolescents report regular heavy episodic drinking. However, only a few German prevention programmes target adolescents of legal drinking age (16 years and above); thus, this trial aims to develop, implement and evaluate 'Klar bleiben' ('Stay clearheaded'), a school-based prevention programme for grade 10 students.

Methods and analysis: 'Klar bleiben' consists of a class commitment to drink responsibly and refrain from hazardous consumption patterns for 9 weeks. The commitment is accompanied by educational lessons on alcohol-related cognitions and consequences. It will be evaluated in a sample of approximately 3000 students (150 classes) from two German federal states (Schleswig-Holstein and Lower Saxony) via a twoarmed cluster randomised controlled trial with baseline and postassessment 6 months apart. The intervention group (75 classes) will participate in 'Klar bleiben', whereas the control group (75 classes) will receive education as usual. 'Klar bleiben' addresses classes, individuals, teachers and parents. It is based on a social norms approach and aims to reduce hazardous drinking and drinking-related consequences in adolescents. Secondary outcomes include general drinking behaviour, use of other substances, alcoholrelated cognitions and social factors. Covariates include sociodemographic characteristics, environmental and individual (vulnerability) factors.

Ethics and dissemination: 'Klar bleiben' provides a multicomponent school-based programme that bridges a gap in alcohol prevention. Similar class-level and social norms-based prevention programmes have already been proven to be successful for other substances among adolescents. Thus, dissemination to other federal states as well as longer term follow-up testing of the robustness of effects is to be anticipated. The study was approved by the Ethics Committee of the German Psychological Society (RH_0620152), and study findings will be disseminated through peerreviewed publications and conference contributions. Trial registration number: German Clinical Trials Register: DRKS00009424.

\section{Strengths and limitations of this study}

- 'Klar bleiben' focuses on older adolescents (aged 16 years and above), who are legally allowed to buy and publicly consume certain alcoholic beverages (beer, wine)

- 'Klar bleiben' addresses four levels (classes, individuals, teachers and parents), and aims to foster social norms that encourage responsible drinking.

- The cluster randomised controlled design accounts for differences between participating individuals, classes and schools.

- All data will be obtained via self-reports, which increase the risk for systematic bias, for example, recall bias, when reporting substance use behaviour.

- The study will be conducted in two federal states, which are not representative of the target age group in Germany, and thus conclusions will be limited.

\section{INTRODUCTION}

There is vast research on the prevalence of alcohol use and abuse among children and adolescents. In their 2014 status report, the WHO found that about $70 \%$ of European adolescents between 15 and 19 years of age drink alcohol on a regular basis, whereas about $31 \%$ practice heavy episodic drinking, also referred to as binge drinking (at least $60 \mathrm{~g}$ of pure alcohol on a single occasion in the last month). ${ }^{1}$ Such drinking behaviour can have harsh consequences; it is associated with academic failure, ${ }^{2}$ cognitive impairment, ${ }^{3}$ physical injuries and sexual risk behaviour ${ }^{4}$ and leads to higher risks for violent incidents and other substance use. ${ }^{5}$ Prospectively, hazardous drinkers have an elevated risk of alcohol misuse and dependence later in life. ${ }^{6}$

Binge drinking or heavy episodic drinking, in particular, often emerges in adolescence: prevalence increases from childhood 
through adolescence and finally peaks in early adulthood (around 21-25 years). Adolescent drinking is often determined by social reasons, for example, belonging to a group or expressing conformity to perceived social norms. ${ }^{78}$ In these social contexts, drinks are often consumed to let loose, to facilitate contact or to enjoy oneself. In recent years, investigation of habits like drinking games, preloading or predrinking has increased. Drinking games connect alcohol intake with a time factor, as a large amount of drinks is consumed in a relatively short amount of time. Preloading or predrinking describes the behaviour of assembling and drinking before social events such as going to a party or a nightclub. ${ }^{9}$

In Germany, wine and beer can legally be bought and publicly consumed at age 16, and spirits and other liquor at age 18. Thus, alcohol prevention for late adolescents (16+ years) in Germany should focus on abstinence, as well as encourage responsible drinking, for example, no binge drinking or heavy episodic drinking, and sobriety in certain situations such as driving, working or being at school. In addition, experimentation with alcohol represents an adolescent developmental task on the way to adulthood-testing one's limits is a normal part of growing up. ${ }^{10}$

Since drinking is often connected to social contexts, the school setting seems to be an ideal place for hazardous alcohol use prevention. Social enhancement or conformity motives, evoked by social situations, are very often linked to hazardous drinking behaviour, underlining the importance of social norms-based approaches. ${ }^{11}$ As of today, there are manifold school-based programmes for alcohol prevention, and there are even some meta-analyses that discern successful programmes from less successful ones. ${ }^{12-16}$ According to these, a successful school-based intervention should: (1) incorporate motivational aspects, (2) focus on peer factors (peer involvement, social norms and peer affiliation) and (3) include social skills and behavioural aspects, for instance, alcohol-related refusal skills.

However, only a few of these prevention programmes target older adolescents ${ }^{14-19}$ and, in addition, most of them are conducted in countries where the purchase and consumption of any alcoholic beverage is illegal for the target population, for example, in the USA. As of today, rigorous evaluations of such programmes, like the Drug Abuse Resistance Education (DARE) programme in the USA, did not show any significant results for relevant alcohol use criteria. ${ }^{18} 19$ However, Brief Alcohol Interventions deliver promising results in short-term and long-term evaluations, ${ }^{14}$ but solely if based on motivational enhancement therapy and if implemented in individual form, suggesting differential effects of brief interventions.

The programme 'Klar bleiben' ('Stay clearheaded'), on the other hand, focuses exclusively on older German adolescents (16+ years), who are legally allowed to buy and consume certain alcoholic beverages. The programme includes class-based educational lessons, a collective class commitment with regard to drinking behaviour, and a lottery with prizes as incentives for classes which finish the intervention successfully.

\section{Hypotheses and aims}

With this study, we want to evaluate the effectiveness of the 'Klar bleiben' prevention programme in grade 10, via a two-arm cluster randomised controlled trial with baseline and postassessment. All measures are listed in table 1 .

\begin{tabular}{|c|c|}
\hline Construct & Measure (acronym) (source) \\
\hline \multicolumn{2}{|l|}{ Gender, age } \\
\hline Migration background & 32 \\
\hline $\begin{array}{l}\text { Socioeconomic status } \\
\text { (parental education) }\end{array}$ & 31 \\
\hline Subjective & Subjective Socioeconomic \\
\hline socioeconomic status & Status scale (SSS-Scale) ${ }^{33}$ \\
\hline $\begin{array}{l}\text { Alcohol (lifetime } \\
\text { prevalence, current } \\
\text { drinking) }\end{array}$ & $34-36$ \\
\hline Binge drinking & 37 \\
\hline Problematic and & Alcohol Use Disorders \\
\hline \multirow[t]{5}{*}{ hazardous alcohol use } & $\begin{array}{l}\text { Identification Test-Consumption } \\
(\text { AUDIT-C) })^{38}\end{array}$ \\
\hline & Brief alcohol screening \\
\hline & $\begin{array}{l}\text { instrument for medical care } \\
(\text { BASIC) })^{39}\end{array}$ \\
\hline & CRAFFT-Screening Test- \\
\hline & German version (CRAFFT-d) ${ }^{40}$ \\
\hline $\begin{array}{l}\text { Consequences of } \\
\text { hazardous drinking }\end{array}$ & Original items ${ }^{41}$ \\
\hline Peer susceptibility & 42 \\
\hline Social norm & Original items ${ }^{35} 43$ \\
\hline $\begin{array}{l}\text { Personality } \\
\text { characteristics }\end{array}$ & Big Five Inventory-10 (BFI-10) ${ }^{44}$ \\
\hline Descriptive norm & 35 \\
\hline $\begin{array}{l}\text { Parents' and siblings' } \\
\text { alcohol use }\end{array}$ & C. \\
\hline Substance use profiles & $\begin{array}{l}\text { Substance Use Risk Profile } \\
\text { Scale (SURPS) }{ }^{45} 46\end{array}$ \\
\hline \multirow[t]{2}{*}{ Drinking motives } & Drinking Motive Questionnaire \\
\hline & $\begin{array}{l}\text { Revised Short Form (DMQ-R } \\
\text { SF) }\end{array}$ \\
\hline Drink refusal & Drink Refusal Self-Efficacy \\
\hline self-efficacy & $\begin{array}{l}\text { Questionnaire-Revised in an } \\
\text { adolescent sample } \\
\text { (DRSEQ-RA) }^{48}\end{array}$ \\
\hline \multirow[t]{2}{*}{ Alcohol expectancies } & Alcohol Expectancy \\
\hline & $\begin{array}{l}\text { Questionnaire-German version } \\
(\text { AEQ-G) }\end{array}$ \\
\hline Class climate & $\begin{array}{l}\text { Youth Self Report-German } \\
\text { version (YSR-G) }\end{array}$ \\
\hline $\begin{array}{l}\text { Bullying and } \\
\text { victimisation }\end{array}$ & Original items ${ }^{35}$ \\
\hline $\begin{array}{l}\text { Use of other } \\
\text { substances }\end{array}$ & Original items \\
\hline
\end{tabular}




\section{Primary outcomes}

The change in hazardous drinking behaviour from baseline to postassessment will primarily be assessed via the change in the frequency of hazardous drinking or binge drinking (cell frequencies and overall mean scores), and via the Alcohol Use Disorders Identification Test-Consumption (AUDIT-C) sum score. In addition, alcohol-related consequences will also be regarded as a primary outcome. They will be assessed via a modified version of the Rutgers Alcohol Problems Index (RAPI; sum score) and the CRAFFT-d (sum score). All of these measures will be applied at baseline and postassessment.

These primary outcomes have been chosen because of their high clinical relevance in the target population. Thus, the hypothesised effects on primary outcomes in the intervention group compared with the control group comprise:

1. A reduction in hazardous drinking behaviour, that is, less binge drinking, less heavy episodic drinking, less frequent drinking.

2. A reduction in alcohol-related consequences, that is, headaches, memory loss, nausea, drunk driving, etc.

To further explore adolescent drinking behaviour and to examine potential confounding variables, the following secondary outcomes and covariates are included.

\section{Secondary outcomes}

General alcohol use (lifetime and current drinking), social factors (peer susceptibility, social norms, estimated descriptive norm), alcohol-related cognitions (drinking motives, drink refusal self-efficacy, alcohol expectancies) and use of other (psychotropic) substances including cigarettes, marijuana and other drugs.

\section{Covariates}

Sociodemographic characteristics (gender, age, (subjective) socioeconomic status, migration background), environmental, school and familial factors (parents' and siblings' alcohol use, class climate, bullying), individual and personality factors (negative thinking, anxiety sensitivity, impulsivity, sensation seeking, Big Five).

\section{METHODS AND ANALYSIS}

"Klar bleiben" was designed by the Institute for Therapy and Health Research in cooperation with the Federal Centre for Health Education (BZgA). The intervention comprises 9 weeks and targets the adolescent population on four levels: class, individual, teacher and parents (figure 1).

\section{Class}

The main component of the intervention is a class commitment with regular monitoring. At the beginning of the intervention, each participating class agrees to refrain from hazardous drinking patterns for the next 9 weeks. This commitment is fixed in a written class contract. Classes that comply with their commitment for the

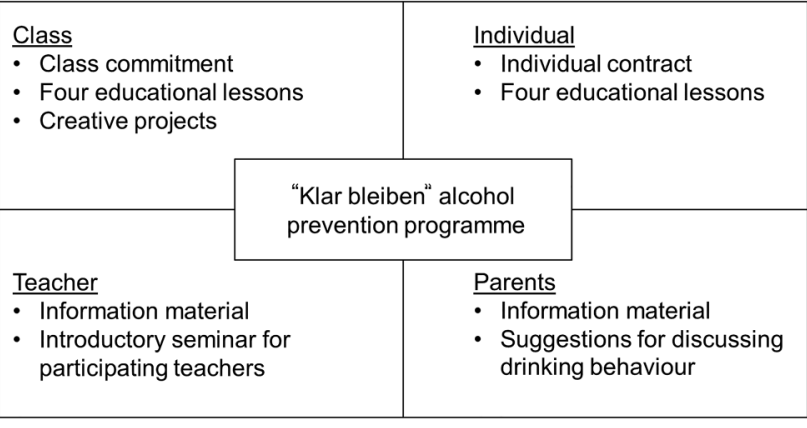

Figure 1 Overview of the intervention's components.

whole 9 weeks are rewarded with attractive prizes in a lottery.

Since compliance is crucial to winning a prize, it is monitored on a regular basis: every fortnight, each student indicates whether he or she has complied with the class commitment or not. These individual reports are counted and reported as class-level frequencies to the project staff. If more than $10 \%$ of a class has not complied, the entire class drops out of the lottery. Moreover, all classes are encouraged to voluntarily submit creative projects dealing with alcohol use in adolescence and its consequences. Classes with the most interesting or innovative submissions will be rewarded with a special prize, independent of their compliance to the initial commitment. Hence, even if a class fails to comply, it may still win a prize.

Besides, each class takes part in up to four educational lessons:

1. Social presence and social norms

The aim is to sensitise students to the presence and connotations of alcohol in modern society and everyday life. Hence, this lesson focuses on factual alcohol use norms, as well as famous aphorisms and examples of alcohol use in society.

2. Advertisements and promotion

Exposure to alcohol-related advertising is associated with increased consumption rates among adolescents. ${ }^{20}$ Moreover, advertisements suggest positive results of alcohol use. Thus, adolescents are encouraged to investigate and reveal hidden messages and strategies in diverse forms of advertising in order to reflect their own behaviour.

\section{Alcohol expectancies}

Alcohol expectancies play an important role in adolescent substance use: positive alcohol expectancies are quite common in this age group, and are linked to earlier onset and problematic drinking. ${ }^{21}{ }^{22}$ Thus, this lesson focuses on personal expectancies, risks and norms associated with (hazardous) alcohol use.

4. Drinking motives

Students are invited to discuss and reflect on their own drinking motives, as well as general drinking motives in adolescence. Examples of alcohol (mis) use will be analysed and discussed and should help the participants to 
clarify their reasons for drinking, and consequently develop and practise adequate coping strategies.

Each lesson takes about $45 \mathrm{~min}$ and will be conducted by the teachers of the participating classes. At the end of the intervention, each teacher is asked to state which lessons were conducted. The educational lessons are not mandatory.

\section{Individual}

In addition to the class commitment, each student receives an individual contract, in which he or she can determine a personal goal for the duration of the intervention. For example, if one was abstinent before, a goal could be to stay abstinent, whereas if one was drinking heavily on the weekends, one could aim to reduce this amount to 1 or 2 drinks an evening. This contract remains private.

\section{Teacher}

The headmaster and teaching staff of each participating school will receive information on the background, material and aims of the programme. Teachers are also invited to comply with the class commitment, and hence represent role models for their students. Additionally, participating teachers will receive an introductory seminar for conducting 'Klar bleiben', suggestions on how to present and implement 'Klar bleiben' in their classes, plus further information on alcohol-related education.

\section{Parents}

Parents will also receive information on the background, material and aims of the programme, and the accompanying assessments. In addition, they will receive suggestions on how to discuss responsible drinking behaviour with their child.

\section{Study design}

This evaluation trial for 'Klar bleiben' is a two-arm two-wave cluster randomised controlled trial. Individual data at each wave are nested at the class and school level. To avoid contamination of experimental conditions, schools are the unit of randomisation. Thus, all grade 10 classes of a participating school are allocated randomly to either the intervention or control group. Classes in the intervention group will take part in the 'Klar bleiben' programme, while the control group receives 'education as usual' without further intervention. Both groups will be surveyed twice, once before (baseline assessment) and once after (postassessment) the intervention. These surveys will be conducted as individual self-reports in each class, administered by trained staff of the Institute for Therapy and Health Research. Process evaluation will be conducted after the intervention: intervention classes will be asked to evaluate the intervention and participating teachers will be asked to evaluate the implementation and the quality of the material and educational lessons and the intervention altogether. The study design and timeline are depicted in figure 2.

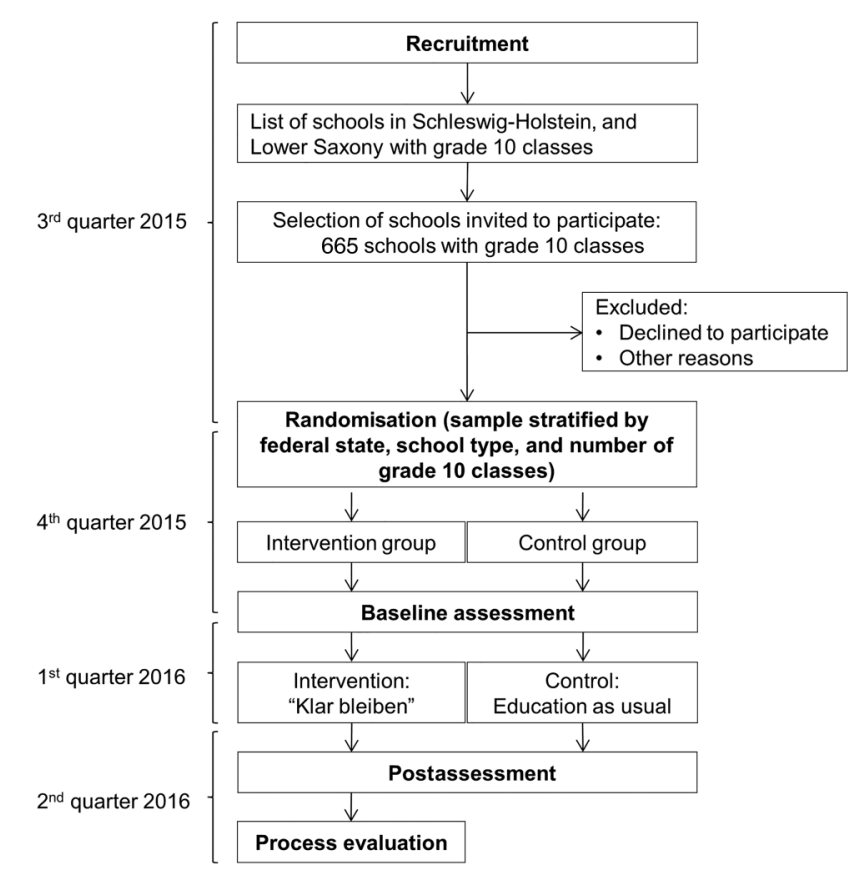

Figure 2 Study design.

\section{Sample size calculation}

One basic assumption in analysing nested data is that data within clusters, for instance, classes or schools, are more similar than between clusters. This clustering effect can statistically be described as $1+(\mathrm{m}-1) \mathrm{p}$, with $m$ referring to the number of units in a cluster, that is, students within a class, and $\mathrm{p}$ to the intraclass correlation coefficient (ICC). ${ }^{23}$ On the basis of prior research, the expected ICC for substance-related outcomes is approximately $0.015 .^{24}$ On average, a class approximately comprises 20 students with parental consent, thus leading to a clustering effect of 1.285. Assuming a significance level of $\alpha=0.05, \quad$ a statistical power of 0.80 and a small-to-medium effect size (Cohen's $\mathrm{d}=0.30$ ) according to meta-analyses of school-based alcohol prevention programmes, ${ }^{12} 151625$ the estimated sample size considering the clustering effect is $\mathrm{N}=2506$ ( $\mathrm{k}=125$ classes). We confirmed this result via a computerised sample size calculator. ${ }^{26}$ Finally, we estimated a dropout rate of $15 \%$, based on previous studies, ${ }^{27} 28$ resulting in a total sample of $\mathrm{N}=3000$ students and $\mathrm{k}=150$ classes. To achieve a sample of this size, a total of 665 schools will be invited to participate.

\section{Procedure and participants}

The target population comprises all 10th graders aged $16+$ years in secondary schools in the federal states of Schleswig-Holstein (SH) and Lower Saxony (LS), Germany. All schools with 10th grade education in SH will be invited $(n=331)$. In addition, a comparable number of schools with grade 10 education from four districts in LS $(n=334)$ will be invited. Headmasters of the respective schools will receive invitational letters, 
information material on background, methods and aims of the study, and copies of the approval letters of the Ethics Committee and their respective Ministry. Schools that agree to participate are obliged to give written consent, and thereby accept the study design and procedure that is, the means of randomisation. Moreover, they will be asked to state the number of grade 10 classes in their school, the names of the respective class teachers and the number of students per class. All grade 10 students of the participating schools will be included in the study. If schools do not wish to participate, they will be asked to explain their reasons for declining. To encourage participation, all participating classes will receive a financial incentive of $100 €$ for participating in both assessments. To conduct non-responder analyses, all invited schools will be asked to provide basic data about school type, size, number of grade 10 classes, number and ethnic composition of student body and number of teachers, as well as the extent of general health education. Participating schools will be stratified by federal state, school type and number of grade 10 classes, and randomly allocated to either the intervention or control group.

Besides, parents (and students 18 years or older) will receive a letter explaining the study. If they give or refuse permission to participate, they signify this by signing a prewritten statement. If participants refuse to take part in the study, their data will not be registered.

\section{Randomisation and stratification}

All participating classes will randomly be assigned to either the control or intervention group (allocation ratio 1:1) via the computational software 'Randomization in Treatment Arms', ${ }^{29}$ which produces a random allocation sequence for stratified randomisation. Schools will be used as the unit of randomisation, in order to avoid interference between the control and intervention groups. Moreover, the sample will be stratified according to the federal state, the type of school and the number of grade 10 classes per school, to balance both groups. The randomisation will be monitored by the principal investigators, and the resulting allocation will be noted.

\section{Data collection, entry and storage}

Schools will be recruited and randomised between September and October 2015. Then the baseline data will be collected in both groups between November and December 2015. The intervention programme 'Klar bleiben' is scheduled for January-March 2016, with the control group simultaneously attending the usual curriculum. Finally, post-test data will be obtained between April and May 2016. A data monitoring committee will not be necessary for two reasons: first, this is not a blinded study, and second, it is a short-term, noninvasive intervention with the opportunity to quit at any time without any negative consequences or side-effects. All data will be entered electronically by trained personnel at the Institute for Therapy and Health Research, where the original forms will be stored. Data entries will be reviewed to check data plausibility and guarantee sufficient data quality. The final data set will be stored for 10 years after study completion. All principal investigators will have access to the final data set. On request, school-level data will be presented to the participating schools as mean scores.

\section{Measures}

Data on federal state and school type will be assessed at the recruitment stage; all other measures will be obtained via anonymous self-report questionnaires. Most measurement tools have previously been tested to assure sufficient reliability and validity. To be able to connect baseline data to post-test data-while guaranteeing anonymity-each student creates an individual seven-digit code. ${ }^{30}$ An overview of all measures for baseline and post-test assessment can be found in table 1 .

\section{Process evaluation}

The quality of implementation may have great impact on the intervention's results; thus, teachers will be asked to rate the implementation of each educational lesson and the intervention altogether via Likert scales. Moreover, participating teachers and students will be asked to provide information about the acceptance and feasibility of the intervention, as well as ideas and suggestions for further improvement. To check the honesty of students in reporting the compliance with their commitment, they will be asked to retrospectively assess their honesty and the honesty of the other participants during the intervention. To identify bias, responses of students with very high and very low scores (via percentiles) will then be compared via Student $t$ tests for independent samples.

\section{Statistical analysis}

Statistical analysis will be conducted with Stata V. $14^{51}$ according to the intention-to-treat paradigm. Program efficacy will be tested via a repeated measurement, nested mixed-effect multilevel regression model with the schools, classes and individuals levels, and random intercepts for these three levels. Experimental group and other covariates will be included as fixed effects. Baseline differences between the intervention and control groups, as well as attrition will be analysed via appropriate tests (for example, $\chi^{2}$, Student $t$ tests for independent samples). Complete case analyses will be performed, unless missing data are high $(>30 \%)$, and data are missing completely at random or missing at random, in which case multiple imputation will be applied.

\section{Ethics and Dissemination}

Schools and parents will be fully informed about the trial. Depending on the federal state, parental consent will be given via active (LS) or passive (SH) agreement. 
Students without parental consent and students who do not want to participate are excluded from all data assessments. Anonymity is guaranteed at all times, because students will only be registered via a self-created individual seven-digit code. Additionally, findings of the current study will be disseminated through peer-reviewed publications and conference contributions.

\section{DISCUSSION}

This study protocol documents the two-arm cluster randomised controlled trial with baseline and postassessment for 'Klar bleiben', a school-based prevention programme for hazardous alcohol use. The programme targets German adolescents in grade 10, aged 16 years and above, who are legally allowed to buy and consume beer and wine in public. Therefore, a major aim of the programme is to promote responsible drinking behaviour. It is expected that participation in the intervention group leads to a reduction in hazardous drinking behaviour (less regular drinking, less heavy drinking) and alcohol-related problems and consequences. To reach this goal, the programme is built on four components that have been empirically linked to adolescent drinking: classes, individuals, teachers and parents.

Although evidence in favour of school-based prevention is mixed for universal prevention programmes, ${ }^{12}$ there are certain aspects that seem to be associated with programme success and that are also incorporated in 'Klar bleiben': social skills and norms, behavioural norms, peer affiliation and motivational enhancement.

Moreover, while most other prevention programmes take place in the classroom but focus on individual experiences with alcohol, 'Klar bleiben' centres on a class-level intervention where the whole group has to stand together to fulfil its commitment and to win a prize. Studies on similar interventions, for example, the Smokefree Class Competition, showed that a class-level design activates a social norm of desirable behaviour-in this case, responsible drinking behaviour-which encourages students to oblige. ${ }^{52-54}$ Additionally, these competitions may promote cohesion within classes, which is positively associated with individual well-being. ${ }^{55}$

Although there are up to four educational lessons, they are not mandatory for participation; therefore, 'Klar bleiben' is not as time-consuming as other programmes and easy to implement for teachers. However, the extent of teacher involvement and integration into regular school life may prove to be an important moderator of programme success. By evaluating the implementation process, such third variable effects are considered.

Nevertheless, a main limitation of this study is the assessment via self-reports. There is an increased risk for systematic bias, such as recall bias, although self-reports have previously been shown to be reliable and valid measures of adolescent drinking. ${ }^{56}{ }^{57}$ Also, since participation is voluntary, non-compliance could become an issue, in particular in groups or classes where a large portion of students report (regular) heavy drinking. Although these classes may drop out of 'Klar bleiben' at an early stage, or they may not be able to win a prize in the lottery, they are still invited to submit creative projects dealing with alcohol use in adolescence, which may lead to secondary treatment effects or at least promising trends. In addition, teachers are encouraged to teach the prepared class courses, regardless of the class commitment, and to invite their students to reflect on their own actions and associated consequences. Subgroup analyses will prove whether there are differential effects depending on the initial level of consumption or the quantity or quality of implementation. Finally, this study will be conducted in two federal German states, which are not representative of the German population. However, if 'Klar bleiben' proves to be successful in reducing hazardous drinking patterns, the programme could be disseminated to other states, and be validated in a larger, nationally representative sample of adolescents, or via long-term follow-up (12+ months) to test the robustness of its effects.

Contributors ST, RH and BI designed the study. ST wrote the first draft of the manuscript, while $\mathrm{RH}$ and $\mathrm{BI}$ revised the manuscript critically for important intellectual content and contributed to the structure. All the authors approved the final version of the manuscript for publication.

Funding This trial is funded by the Federal Centre for Health Education $(B Z g A)$ on behalf of the German Ministry of Health. All authors received grants from the Federal Centre for Health Education, on behalf of the German Ministry of Health, during the conduct of the study.

\section{Competing interests None.}

Ethics approval This study has been approved by the Ethics Committee of the German Psychological Society and the Ministries of Education of Schleswig-Holstein and Lower Saxony.

Provenance and peer review Not commissioned; peer reviewed for ethical and funding approval prior to submission.

Data sharing statement The trial data set and any additional unpublished data will only be available to research staff at the Institute for Therapy and Health Research (IFT-Nord) who initially conducted the study.

Open Access This is an Open Access article distributed in accordance with the Creative Commons Attribution Non Commercial (CC BY-NC 4.0) license, which permits others to distribute, remix, adapt, build upon this work noncommercially, and license their derivative works on different terms, provided the original work is properly cited and the use is non-commercial. See: http:// creativecommons.org/licenses/by-nc/4.0/

\section{REFERENCES}

1. World Health Organisation. Global status report on alcohol and health 2014. Geneva: WHO, 2014.

2. Kelly AB, Evans-Whipp TJ, Smith R, et al. A longitudinal study of the association of adolescent polydrug use, alcohol use and high school non-completion. Addiction 2015;110:627-35.

3. Tapert SF, Granholm E, Leedy NG, et al. Substance use and withdrawal: neuropsychological functioning over 8 years in youth. $J$ Int Neuropsychol Soc 2002;8:873-83.

4. Bonomo $\mathrm{Y}$, Coffey $\mathrm{C}$, Wolfe R, et al. Adverse outcomes of alcohol use in adolescents. Addiction 2001;96:1485-96.

5. Miller JW, Naimi TS, Brewer RD, et al. Binge drinking and associated health risk behaviors among high school students. Pediatrics 2007;119:76-85.

6. Andersen A, Due P, Holstein BE, et al. Tracking drinking behaviour from age 15-19 years. Addiction 2003;98:1505-11.

7. Kuntsche E, Knibbe R, Gmel G, et al. Why do young people drink? A review of drinking motives. Clin Psychol Rev 2005;25:841-61. 
8. Kuntsche E, Knibbe R, Gmel G, et al. Who drinks and why? A review of socio-demographic, personality, and contextual issues behind the drinking motives in young people. Addict Behav 2006:31:1844-57.

9. Wahl S, Sonntag T, Roehrig J, et al. Characteristics of predrinking and associated risks: a survey in a sample of German high school students. Int J Public Health 2013;58:197-205.

10. Havighurst RJ. Human development and education. New York: Longmans \& Green, 1953.

11. Kuntsche E, Wicki M, Windlin B, et al. Drinking motives mediate cultural differences but not gender differences in adolescent alcohol use. J Adolesc Health 2015;56:323-9.

12. Foxcroft DR, Tsertsvadze A. Universal school-based prevention programs for alcohol misuse in young people. Cochrane Database Syst Rev 2011;(5):CD009113.

13. Gottfredson DC, Wilson DB. Characteristics of effective school-based substance abuse prevention. Prev Sci 2003;4:27-38.

14. Hennessy EA, Tanner-Smith EE. Effectiveness of brief school-based interventions for adolescents: a meta-analysis of alcohol use prevention programs. Prev Sci 2014;16:463-74.

15. Strøm H, Adolfsen F, Fossum S, et al. Effectiveness of school-based preventive interventions on adolescent alcohol use: a meta-analysis of randomized controlled trials. Subst Abuse Treat Prev Policy 2014;9:48

16. Tobler NS, Roona MR, Ochshorn P, et al. School-based adolescent drug prevention programs: 1998 meta-analysis. J Prim Prev 2000;20:275-336.

17. Bailey KA, Baker AL, Webster RA, et al. Pilot randomized controlled trial of a Brief Alcohol Intervention group for adolescents. Drug Alcohol Rev 2004;23:157-66.

18. Ennett ST, Tobler NS, Ringwalt CL, et al. How effective is Drug Abuse Resistance Education? A meta-analysis of Project DARE outcome evaluations. Am J Public Health 1994;84:1394-401.

19. Ennett ST, Rosenbaum DP, Flewelling RL, et al. Long-term evaluation of Drug Abuse Resistance Education. Addict Behav 1994:19:113-25.

20. Morgenstern M, Isensee B, Sargent JD, et al. Exposure to alcohol advertising and teen drinking. Prev Med 2011;52:146-51.

21. Jester JM, Wong MM, Cranford JA, et al. Alcohol expectancies in childhood: change with the onset of drinking and ability to predict adolescent drunkenness and binge drinking. Addiction 2015;110:71-9.

22. Newton NC, Barrett EL, Swaffield L, et al. Risky cognitions associated with adolescent alcohol misuse: moral disengagement, alcohol expectancies and perceived self-regulatory efficacy. Addict Behav 2014;39:165-72.

23. Murray DM, Varnell SP, Blitstein JL. Design and analysis of group-randomized trials: a review of recent methodological developments. Am J Public Health 2004;94:423-32.

24. Faggiano $\mathrm{F}$, Richardson $\mathrm{C}$, Bohrn $\mathrm{K}$, et al. A cluster randomized controlled trial of school-based prevention of tobacco, alcohol and drug use: the EU-Dap design and study population. Prev Med 2007:44:170-3.

25. Sussman S, Arriaza B, Grigsby TJ. Alcohol, tobacco, and other drug misuse prevention and cessation programming for alternative high school youth: a review. J Sch Health 2014;84:748-58.

26. Campbell MK, Thomson S, Ramsay CR, et al. Sample size calculator for cluster randomized trials. Comput Biol Med 2004;34:113-25.

27. Hanewinkel R, Sargent JD. Longitudinal study of exposure to entertainment media and alcohol use among German adolescents. Pediatrics 2009;123:989-95.

28. Hanewinkel R, Isensee B, Sargent JD, et al. Cigarette advertising and teen smoking initiation. Pediatrics 2011:127:e271-8.

29. Pahlke F, König IR, Ziegler A. Randomization in Treatment Arms (RITA): Ein Randomisierungs-Programm für klinische Studien [A randomization program for clinical trials]. Inform Biom Epidemiol Med Biol 2004;35:1-22.

30. Galanti MR, Siliquini R, Cuomo L, et al. Testing anonymous link procedures for follow-up of adolescents in a school-based trial: the EU-DAP pilot study. Prev Med 2007;44:174-7.

31. Suchert V, Isensee B, Hansen J, et al. "läuft."-a school-based multi-component program to establish a physically active lifestyle in adolescence: study protocol for a cluster-randomized controlled trial. Trials 2013;14:416.

32. Statistisches Bundesamt. Zensus 2011 - Methoden und Verfahren [Census 2011 - methods and measures]. Wiesbaden: Statistisches Bundesamt, 2015.

33. Hoebel J, Müters S, Kuntz B, et al. Messung des subjektiven sozialen Status in der Gesundheitsforschung mit einer deutschen Version der MacArthur Scale [Measuring subjective social status in health research with a German version of the MacArthur Scale]. Bundesgesundheitsblatt 2015;58:749-57.

34. Donovan JE, Molina BS. Types of alcohol use experience from childhood through adolescence. J Adolesc Health 2013;53:453-9.

35. Hansen J, Hanewinkel R, Maruska K, et al. The 'Eigenständig werden' prevention trial: a cluster randomised controlled study on a school-based life skills programme to prevent substance use onset. BMJ Open 2011;1:e000352.

36. Reboussin BA, Song EY, Shrestha A, et al. A latent class analysis of underage problem drinking: evidence from a community sample of 16-20 year olds. Drug Alcohol Depend 2006;83:199-209.

37. Wechsler H, Nelson TF. Binge drinking and the American college student: what's five drinks? Psychol Addict Behav 2001;15:287-91.

38. Rumpf HJ, Wohlert T, Freyer-Adam J, et al. Screening questionnaires for problem drinking in adolescents: performance of AUDIT, AUDIT-C, CRAFFT and POSIT. Eur Addict Res 2013;19:121-7.

39. Bischof G, Reinhardt S, Grothues J, et al. Development and evaluation of a screening instrument for alcohol-use disorders and at-risk drinking: the brief alcohol screening instrument for medical care (BASIC). J Stud Alcohol Drugs 2007;68:607-14.

40. Tossmann $\mathrm{P}$, Kasten L, Lang $\mathrm{P}$, et al. Bestimmung der konkurrenten Validität des CRAFFT-d: Ein Screeninginstrument für problematischen Alkoholkonsum bei Jugendlichen [Determination of the concurrent validity of the CRAFFT-d: a screening instrument for problematic alcohol consumption]. Z Kinder Jugendpsychiatr Psychother 2009;37:451-9.

41. White HR, Labouvie EW. Towards the assessment of adolescent problem drinking. J Stud Alcohol 1989;50:30-7.

42. Pierce JP, Choi WS, Gilpin EA, et al. Validation of susceptibility as a predictor of which adolescents take up smoking in the United States. Health Psychol 1996;15:355-61.

43. Needle R, McCubbin $\mathrm{H}$, Wilson $\mathrm{M}$, et al. Interpersonal influences in adolescent drug use - the role of older siblings, parents, and peers. Int J Addict 1986;21:739-66.

44. Rammstedt B, John OP. Measuring personality in one minute or less: a 10-item short version of the Big Five Inventory in English and German. J Res Pers 2007:41:203-12.

45. Paul S, Rodehacke S, Krömer NB. Die deutsche Version der Substance Use Risk Profile Scale (SURPS): psychometrische validierung anhand von Deutschen, Englischen, Irischen und Französischen Jugendlichen [German version of the Substance Use Risk Profile Scale (SURPS) psychometric validation in a sample of German, English, Irish, and French adolescents]. Suchttherapie 2013;14:29.

46. Woicik PA, Stewart SH, Pihl RO, et al. The Substance Use Risk Profile Scale: a scale measuring traits linked to reinforcement-specific substance use profiles. Addict Behav 2009;34:1042-55.

47. Kuntsche E, Kuntsche S. Development and validation of the Drinking Motive Questionnaire Revised Short Form (DMQ-R SF) $J$ Clin Child Adolesc Psychol 2009;38:899-908.

48. Young RM, Hasking PA, Oei TP, et al. Validation of the Drinking Refusal Self-Efficacy Questionnaire-Revised in an Adolescent Sample (DRSEQ-RA). Addict Behav 2007;32:862-8.

49. Demmel R, Hagen J. Faktorenstruktur und psychometrische Eigenschaften einer gekürzten deutschsprachigen Version des Alcohol Expectancy Questionnaire (Brief AEQ-G). [Factor Structure and Psychometric Properties of a Brief German Version of the Alcohol Expectancy Questionnaire (Brief AEQ-G)]. Z Differ Diagn Psychol 2002;23:205-16.

50. Walter R, Remschmidt H, Deimel W. Reliabilität und Validität der deutschen Version des Youth Self Report [Reliability and validity of the German version of the Youth Self Report]. Z Kinder Jugendpsychiatr 1994;22:23-38.

51. StataCorp. Stata Statistical Software: Release 14. College Stations, Texas: StataCorp LP, 2015

52. Isensee B, Morgenstern M, Stoolmiller M, et al. Effects of Smokefree Class Competition 1 year after the end of intervention: a cluster randomised controlled trial. J Epidemiol Community Health 2012;66:334-41.

53. Isensee $B$, Hanewinkel R. Meta-analysis on the effects of Smoke-free Class Competition on smoking prevention in adolescents. Eur Addict Res 2012;18:110-15.

54. Wiborg G, Hanewinkel R. Effectiveness of the "Smoke-Free Class Competition" in delaying the onset of smoking in adolescence. Prev Med 2002;35:241-9.

55. Gergana M, John TP. Cohesion and individual well-being of members in self-managed teams. Leadersh Org Dev J 2014;35:429-41.

56. Del Boca FK, Darkes J. The validity of self-reports of alcohol consumption: state of the science and challenges for research. Addiction 2003;98(Suppl 2):1-12.

57. Lintonen T, Ahlström S, Metso L. The reliability of self-reported drinking in adolescence. Alcohol Alcohol 2004;39:362-8. 